\title{
Doped Amorphous Carbon Films Prepared by Liquid Phase Electrodeposition
}

\author{
Canyan Che, Yang Li, Guifeng Zhang*, Dewei Deng \\ Key Laboratory for Materials Modification by Laser, Ion and Electron Beams, Ministry of Education, \\ School of Materials Science and Engineering, Dalian University of Technology, Dalian, China \\ Email: *gfzhang@dlut.edu.cn
}

Received September 22, 2013; revised October 30, 2013; accepted November 21, 2013

Copyright (C) 2014 Canyan Che et al. This is an open access article distributed under the Creative Commons Attribution License, which permits unrestricted use, distribution, and reproduction in any medium, provided the original work is properly cited. In accordance of the Creative Commons Attribution License all Copyrights (c) 2014 are reserved for SCIRP and the owner of the intellectual property Canyan Che et al. All Copyright @ 2014 are guarded by law and by SCIRP as a guardian.

\begin{abstract}
It has theoretical significance and practical value to synthetize and modify amorphous carbon films by liquid electro-deposition technique due to its low cost, simple equipment, and better operability in uniform deposition of the films with large-area and complex shape work pieces. This article introduces the research situation of the carbon films prepared by liquid phase electrochemical deposition according to the applied voltage, discusses the influence of experimental parameters on the film properties, and describes possible reaction mechanisms. It summarizes the research progress of amorphous carbon films doped with metal and nonmetals. Finally, existing problems have been demonstrated and suggestions on research hotspots in the future are given.
\end{abstract}

\section{KEYWORDS}

\section{Liquid Electrodeposition; Doping; Carbon Films}

\section{Introduction}

Amorphous carbon films have an extensive application prosperity in the mechanical, electronic, chemical, military and aerospace fields due to their low friction coefficient, good wear resistance, chemical inertness, and high transmittance in a wide range from infrared to ultraviolet. Recently, various physical vapor deposition (PVD) and chemical vapor deposition (CVD) methods, including ion beam deposition, pulsed laser deposition, and plasma enhanced CVD, etc., are used to prepare the films. However, the deficiencies of these techniques are more complex equipment, higher cost, and more difficult in deposition uniform films with large-area and irregular surface [1]. Maissel et al. [2] pointed out that the film materials prepared in the gas phase can be also obtained from electrochemical deposition in the liquid phase, and vice versa. Hydrogenated carbon films were successfully synthesized by Namb [3] in 1992 from electrochemical deposition technique. Generally, using a high voltage, the films were deposited on substrate as cathode and graphite or $\mathrm{Pt}$

${ }^{*}$ Corresponding author. served as anode $[4,5]$. The report has attracted much attention in view of the facts of low-cost, random choice of substrates and carbon sources, and easy doping.

\section{Amorphous Carbon Films Prepared by Cathodic Deposition Mode}

\subsection{Application of High-Voltage (1000 - 3000 V)}

Some experiments have proved that hydrogenated amorphous carbon films with obvious characteristics of diamond structure can be prepared using liquid-phase deposition under atmospheric pressure and low temperature. Figure 1 shows SEM photographs of the films deposited from organic solvents of methanol, ethanol, and isopropyl alcohol. These organic solvents possesses a lower electrical conductivity and hence a higher voltage in the range of 1000 - $3000 \mathrm{~V}$ should be applied between two electrodes. All the same, the deposition rate is very low.

Two electrodes can be regarded as idea parallel plate capacitor. Solution with a dielectric constant $\varepsilon$ is polarized under the action of external electric field. Induced charges created on the electrodes and the density (also 


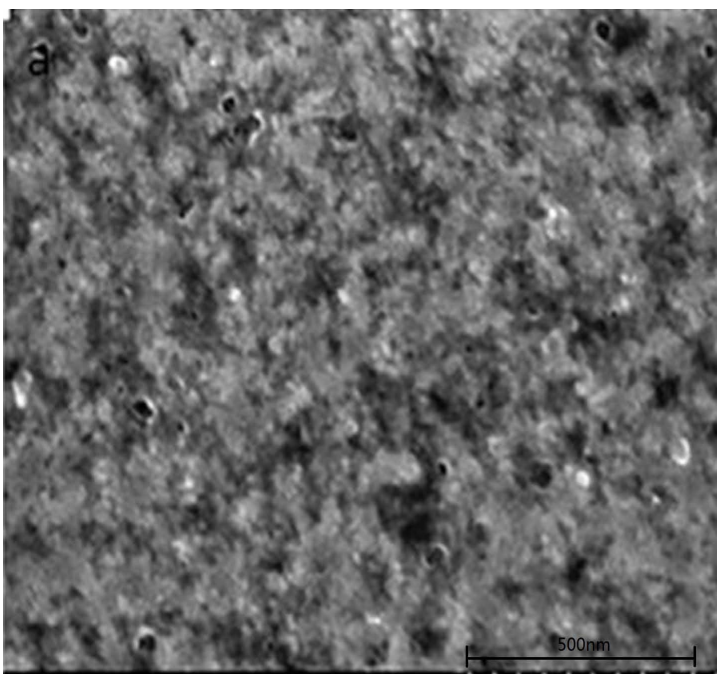

(a)

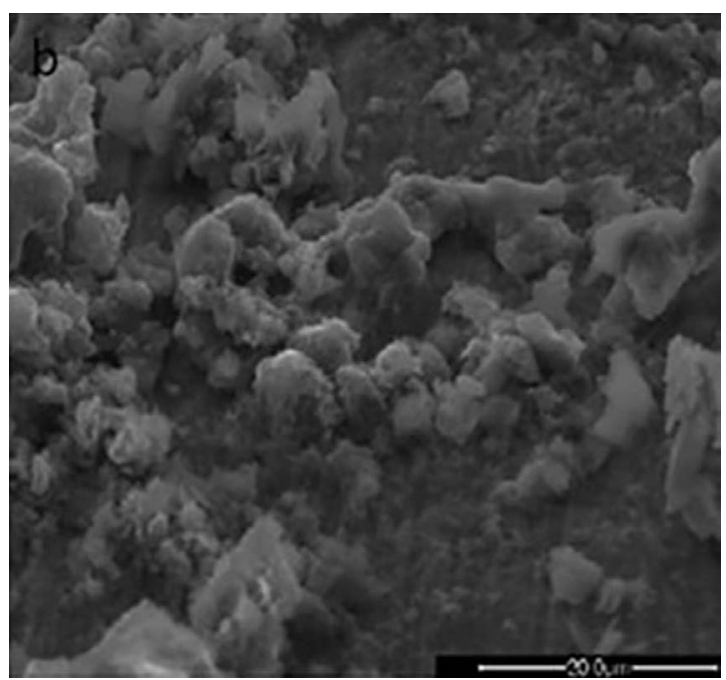

(b)

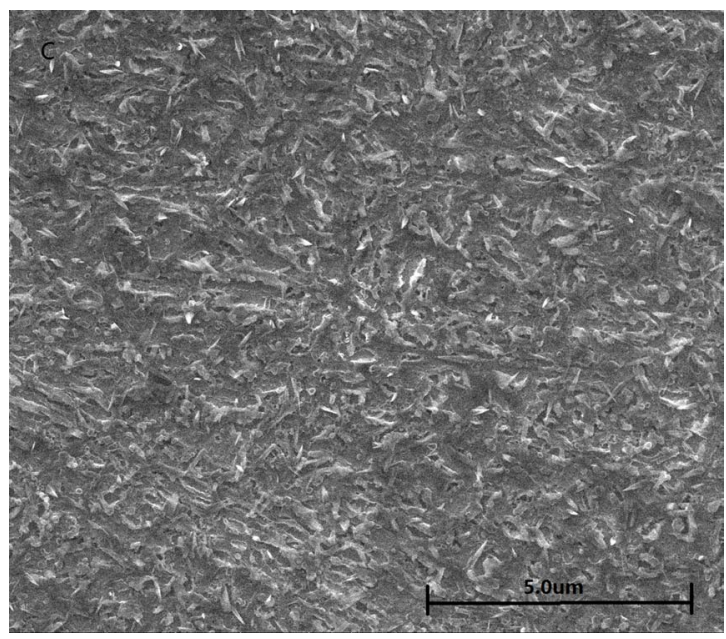

(c)

Figure 1. SEM photographs of the films depositd from various organic solvents of (a) methanol [6]; (b) ethanol [7], and (c) is opropyl alcohol [8] at $1000 \mathrm{~V}$. known as the polarization ability) is determined by the formula $P=\varepsilon_{0}(\varepsilon-1) E$, where $\varepsilon_{0}$ is constant and $E$ electric field intensity. Obviously, under the same deposition conditions $P$ depends only on the dielectric constants. It is therefore necessary to select organic solutions with high dielectric constant [9]. Carbon sources frequently used contain methanol [10], alcohol [11,12], methyl cyanide, DMF, DMSO [13,14], and so on. The growth mechanism of carbon membrane can be briefly described as follows: 1) the center of positive and negative charges in polarized molecules is non-coincidence and electronic distribution tends to the polar functions. Under the action of a high external electric field, the charges are more away from the center, molecules will be induced polarization; 2) positively and negatively charged polar functional groups generate in the solution by interrupting weak chemical bonds; 3) meanwhile, the surface of the electrodes is activated, and the charged particles move to the electrode and are captured on the activity locations; 4) the positively charged groups are captured and obtains the extra electron on the cathode, and then dehydrogenation and deoxidization reactions occur to form C-C bonds [15]. It is also necessary for a ideal reactant to have a low viscosity coefficient for the ideal reactants. If the viscosity coefficient of electrolyte is too high, negatively charged ions are difficult to move away from the interfacial reaction zone during reaction, which will lead to the accumulation of more and more anions near the cathode. As a result, an electric layer is formed in the contact zone between the substrate and the organic reagent. The electric field in the electrical double layer will offset the outfield, making polarization and bond breaking in the region difficult, which is not conducive to the growth of carbon films. Basic properties of commonly used carbon sources are shown in Table 1.

\subsection{Application of Mid-Voltage (200 - $800 \mathrm{~V})$}

Inspired by atomic hydrogen effectively etching graphite phase in vapor deposition, we attempted to add deionized water into organic reagent. The results showed the deposition voltage reduced or growth rate increased and the

Table 1. Basic properties of commonly used carbon sources.

\begin{tabular}{cccc}
\hline Solution & $\begin{array}{c}\text { Dielectric } \\
\text { constant }\end{array}$ & $\begin{array}{c}\text { Viscosity coefficient } \\
(\mathrm{mPa} \cdot \mathrm{S})\end{array}$ & Dipole moment \\
\hline Methanol & $33.0^{20}$ & $0.544^{25}$ & 1.70 \\
Ethanol & $25.3^{20}$ & $1.074^{25}$ & 1.69 \\
Acetic acid & $6.20^{20}$ & $1.056^{25}$ & 1.70 \\
DMF & $38.25^{20}$ & $0.794^{25}$ & 3.82 \\
Methyl cyanide & $36.64^{20}$ & $0.329^{30}$ & 3.924 \\
Acetone & $21.0^{20}$ & $0.306^{25}$ & 2.88 \\
\hline
\end{tabular}

The superscripts refer to the measuring temperature and datas refer to reference [16]. 
sp3 content in the films increased [17], and even nanodiamond particles have been found in the deposited films.

We proposed a thermodynamic coupling model to explain the above phenomenon [18]. The growth process of carbon film can be divided into two steps: 1) organic molecules are decomposed into the groups containing carbon and there are captured by the electrode, 2) carbon films grow through a series of reactions. Using methanol as an example, carbon films are grown by generating methyl and occurring dehydrogenation conversion. The procedure is simply expressed as follows

$$
\begin{gathered}
\mathrm{CH}_{3} \mathrm{OH} \rightarrow \mathrm{CH}_{3}+\mathrm{OH} \quad \Delta G_{1}>0 \\
(T=300 \mathrm{~K}, P=101 \mathrm{KPa}) \\
\mathrm{CH}_{3} \rightarrow \mathrm{C}\left(\mathrm{sp}^{3}, \mathrm{sp}^{2}\right)+\frac{3}{2} \mathrm{H}_{2} \quad \Delta G_{2}<0 \\
(T=300 \mathrm{~K}, P=101 \mathrm{KPa})
\end{gathered}
$$

where $\Delta G$ is the change of Gibbs free energy. The increment of Gibbs free energy is a criterion for judging whether a reaction occurspontaneously at constant temperature and constant pressure. $\Delta G_{1}>0$ means the reaction (1) to the right with energy input and $\Delta G_{2}<0$ means the methyl in thermodynamics can spontaneously translate into carbon and hydrogen. Note that this reaction usually needs to overcome an energy barrier, otherwise the resultant may be metastable phase, such as ethylene chain etc. $\mathrm{H}_{2} \mathrm{O}$ can be easily decomposed into $\mathrm{H}$ ion and hydroxyl by inputting a lower energy, that is, lower voltage because of its strong polarity. $\mathrm{H}$ ion obtains electron to change into atomic hydrogen. Hydrogen atoms near the cathode should have the following two functions: interrupting the $\mathrm{C}-\mathrm{H}$ bonds in the methyl to form C-C bond, which is better for increasing growth rate, and breaking chemical bonds in organic molecules owing to large amounts of energy from the association of hydrogen atoms.

$$
\mathrm{H} \cdot \rightarrow 0.5 \mathrm{H}_{2} \quad \Delta G_{3}<0(T=300 \mathrm{~K}, P=101 \mathrm{KPa})(3)
$$

It is believed that three reactions (1), (2), and (3) could be thermodynamic coupled when the above two processes occur at the same time. The overall coupling reaction is expressed as follows:

$$
\begin{array}{r}
\mathrm{CH}_{3} \mathrm{OH}+x \mathrm{H} \cdot=\mathrm{C}\left(\mathrm{sp}^{3}\right)+\mathrm{OH}+(0.5 x+1.5) \mathrm{H}_{2} \\
(T=300 \mathrm{~K}, P=101 \mathrm{KPa}) \\
\Delta G_{4}=\Delta G_{1}+\Delta G_{2}+x \Delta G_{3}
\end{array}
$$

Here $x$ represents the coupling coefficient, increased with concentration of hydrogen atoms near the substrate. The larger the coupling coefficient $x$, the smaller $\Delta G_{4}$, the reaction (4) is more easily to right. Therefore, the growth of carbon films can occur under a low voltage at which water molecules can be polarized and decomposed.

\subsection{Application of Low Voltage}

Attempts had been made by others to prepare amorphous carbon films using aqueous solution of acetic acid or formic acid as electrolyte $[19,20]$ under the pressure less than $20 \mathrm{~V}$. This result is easy to be explained based on the above discussion because hydrogen ions can be spontaneously generated from the ionization of aqueous solution of acetic acid or formic acid. Therefore, the reaction (4) can carry out to right only applying a low voltage. That means a large amount of free ion hydrogen in liquid phase guarantees to grow amorphous carbon films under low external voltage. Effect of voltage on the carbon film growth can be summarized in Table 2 .

\section{Amorphous Carbon Films Prepared by Anodic Deposition Mode}

So far only a few researchers attempt to prepare carbon films by anode deposition mode. A brittle carbon film has been successfully synthesized on the anode in 1997 by Novikov et al. [21], using anodic oxidation of aqueous solution of ammonia acetylene under 2.0 - 3.0 V. Meanwhile, in 2001 they gained nano-diamond particles chosing $50 \mathrm{~mol} \%$ ammonium acetate in acetic acid solution as electrolyte at the anodic basement [22]. In other reports such as Shevchenko [23] and Li [24] et al. $\mathrm{C}_{2} \mathrm{HLi} / \mathrm{DMSO}$ and methanol were used as solutions.

\subsection{Amorphous Carbon Films Prepared by Anodic Deposition Mode}

It is generally believed that the only chemical bonds with lowest energy, for example, methyl and hydroxyl in $\mathrm{CH}_{3} \mathrm{OH}$, can be interrupted under high voltage. However, we think the bond polarity is equally important in addition to bond energy. The bond with a stronger polarity or bigger electronagativity difference is more easily interrupted. Bond energy and electronegativity difference of typical chemical bonds are shown in Table 3.

The bond energies of three types of bonds in methanol, $\mathrm{H}-\mathrm{CH}_{2} \mathrm{OH}, \mathrm{CH}_{3} \mathrm{O}-\mathrm{H}$, and $\mathrm{CH}_{3}-\mathrm{OH}$, are $411 \mathrm{~kJ} \cdot \mathrm{mol}^{-1}$,

Table 2. Effect of voltage on the carbon film growth.

\begin{tabular}{ccc}
\hline Electrolyte & Voltage values & Voltage function \\
\hline Pure organic reagents & $>1200 \mathrm{~V}$ & $\begin{array}{c}\text { Polarization and decomposition } \\
\text { of organic reagents }\end{array}$ \\
$\begin{array}{c}\text { Organic reagents } \\
\text { with deionized water }\end{array}$ & $200 \mathrm{~V}-800 \mathrm{~V}$ & $\begin{array}{c}\text { Polarization and decomposition } \\
\text { of deionized water }\end{array}$ \\
$\begin{array}{c}\text { Electrolyte with a } \\
\text { large amount of H } \\
\text { ions }\end{array}$ & $<20 \mathrm{~V}$ & Trapping hydrogen ions \\
\hline
\end{tabular}


Table 3. Bond energy and electronegativity difference of typical chemical bonds.

\begin{tabular}{ccccc}
\hline Chemical bond & C-C & C-O & C-H & O-H \\
\hline Bond energy $(\mathrm{kJ} / \mathrm{mol})$ & 346 & 358 & 411 & 459 \\
Electronegativity difference & 0 & 1 & 0.4 & 1.4 \\
\hline
\end{tabular}

$459 \mathrm{~kJ} \cdot \mathrm{mol}^{-1}$, and $358 \mathrm{~kJ} \cdot \mathrm{mol}^{-1}$, respectively. It is clear that $\mathrm{C}-\mathrm{O}$ bond possesses a lowest energy and moderate electronegativity, and both the bond energy and electronegativity of the $\mathrm{O}-\mathrm{H}$ bond are largest. Therefore, interruption of both bonds of $\mathrm{C}-\mathrm{O}$ and $\mathrm{O}-\mathrm{H}$ will occur in a strong electric field:

$\mathrm{CH}_{3} \mathrm{OH} \rightarrow \mathrm{CH}_{3} \mathrm{O}^{\delta-}+\mathrm{H}^{\delta+}$ and

$\mathrm{CH}_{3} \mathrm{OH} \rightarrow \mathrm{CH}_{3}^{\delta+}+\mathrm{OH}^{\delta-}$. The radicals $\mathrm{CH}_{3} \mathrm{O}^{\delta-}$ move to anode to form amorphous carbon film by a series of dehydrogenation and deoxidization reactions.

\subsection{Effect of Deionized Water}

In contrast, we have also investigated the influence of deionized water on growth of carbon films on anode. Silicon wafer is elected for the substrate to prevent the anode oxidation and the deposition voltage is kept at 800 $\mathrm{V}$. Infrared spectra of the films deposited from various concentration methanol in water are shown in Figure 2. It can be seen that the films contain large number of $\mathrm{O}-\mathrm{H}$ bonds and its content increases with the increase of deionized water, so also for the O-Si-O, which demonstrates that the substrate as anode is easy to be oxidized. $\mathrm{OH}^{-}$ ions generated by electrolyzing water captured on the anode are not conductive to carbon film growth on the anode, which is different with cathodic deposition mode.

\section{Doped Carbon Films Prepared by Liquid Phase Method}

\subsection{Thin Films Doped with Metal}

Microhardness, tribological property, adhesive strength, chemical stability, and electrical conductivity of the films can be generally enhanced by doping metals [26]. Compared with the gas phase method, doping metals into the carbon films becomes much easier for liquid phase deposition technique. Amorphous carbon films doped with metal have been successfully obtained using metal salt solution as electrolyte or nanoparticles as dopant.

\subsubsection{Metal Salt Electrolyte}

Wan et al. [27] prepared diamond-like carbon film at $1200 \mathrm{~V}$ and $55^{\circ} \mathrm{C}-60^{\circ} \mathrm{C}$ for $10 \mathrm{~h}$ using methanol as carbon source and ferric acetylacetonate as dopant. The results showed that $10 \%$ doped Fe with preferred orientation distributed in the DLC matrix and the sp3 content and the electrical conductivity were enhanced. It is well
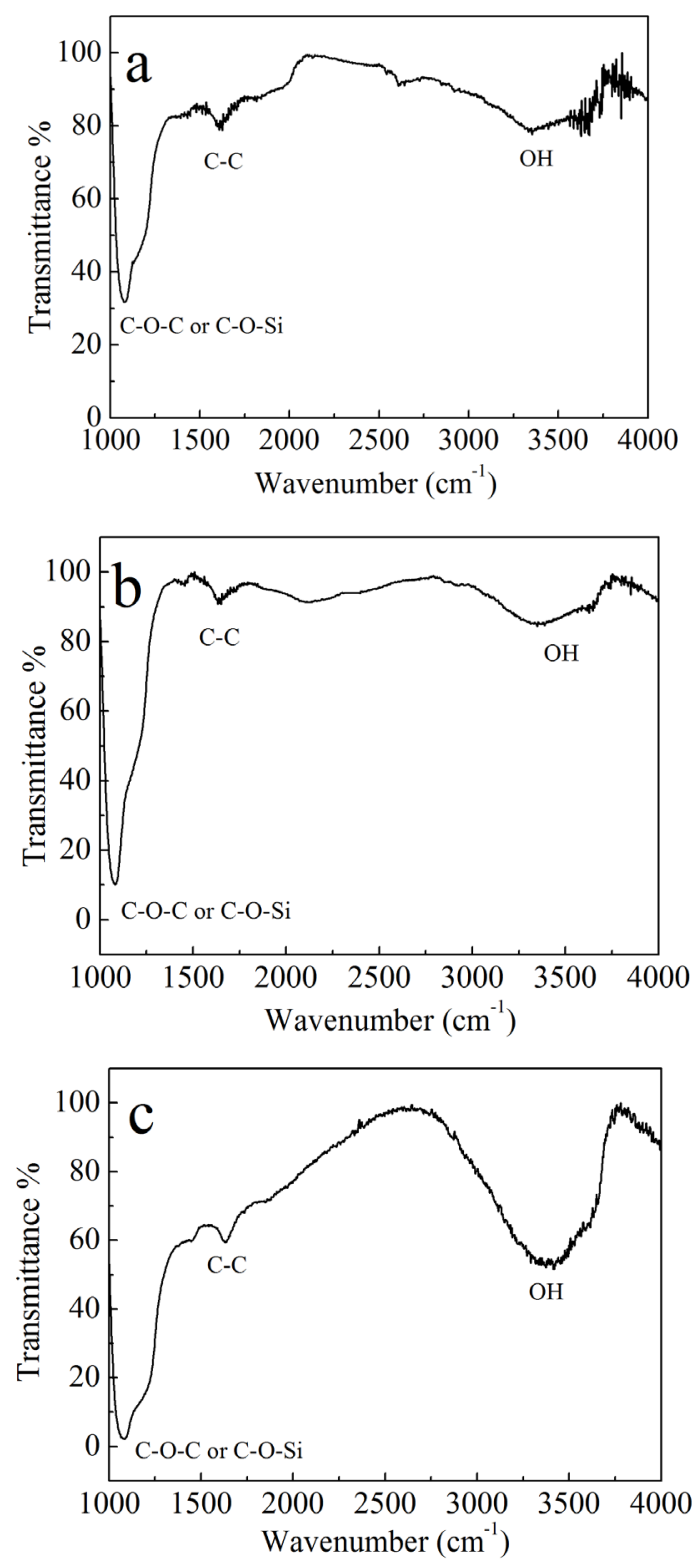

Figure 2. IR spectrogram of the deposited carbon films from volume ratio of $\mathrm{H}_{2} \mathrm{O}$ to $\mathrm{CH}_{3} \mathrm{OH}$ of (a) 0 ; (b) 1; and (c) 2. [25].

understood that methyl $\mathrm{CH}^{+}$and $\mathrm{Fe}$ ion $\mathrm{Fe}^{3+}$ move to cathode to form the Fe-doped DLC film under high voltage.

A brown Cu-doped DLC film can also be deposited at $1600 \mathrm{~V}$ and $60^{\circ} \mathrm{C}$ using from methyl cyanide as carbon source and $\left[\mathrm{Cu}\left(\mathrm{CH}_{3} \mathrm{CN}\right)_{4}\right] \mathrm{ClO}_{4}$ as dopant [28], where the solubility of copper salt solution, current density, and deposition time are $1.5 \mathrm{mM}, 2.0-5.5 \mathrm{~mA} \cdot \mathrm{cm}^{-2}$, and 20 $\mathrm{min}$, respectively. It was said that the sp3 content in the film was increased to a certain extent. In order to achieve a co-deposition of $\mathrm{Cu}$ and carbon, dimethylsulfoxide (DMSO) with a large dipole moment and dielectric constant was selected as carbon source [29]. 
Carbon films doped metals can be also achieved by anode deposition mode. A light yellow $\mathrm{Ru} / a-\mathrm{CN} x$ : $\mathrm{H}$ film with a $\mathrm{Ru} / \mathrm{C} / \mathrm{N}$ atomic ratio of $0.28 / 0.33 / 1$ has be synthesized using a mix solution of $\mathrm{Ru}_{3}(\mathrm{CO})_{12}$ and methyl cyanide at a voltage of $1200 \mathrm{~V}$ and a temperature of $55^{\circ} \mathrm{C}$ for a deposition time of $10 \mathrm{~h}$. The nanoruthenium particles with (101) preferred orientation were not distributed evenly throughout the doped film. Nevertheless, the resistivity of the Ru-doped film decreased greatly from $108 \Omega \cdot \mathrm{m}$ to $100 \Omega \cdot \mathrm{m}$. Yu et al. [30] used cathode deposition mode instead to prepare Ru-doped amorphous carbon films, containing the evenly distributed nanoRuthenium particles with a diameter range of $2-4 \mathrm{~nm}$. Ma et al. [31] claimed that doping Ni can improve adhesive strength and wear resistance. Experiments indicate that it is also easy to realize co-doping of metals (for example, $\mathrm{Cu}$ and $\mathrm{Ag}$ [32]) in electrochemical deposition.

\subsubsection{Metal Nanoparticles as Dopant}

In fact it is difficult to control the co-deposition of metal and carbon because of an obvious difference in ionization properties between metal salts and organic solvents. Consequently, some researchers use metal nanoparticles as dopant. During the deposition process electromagnetic stirring should be essential to ensure uniformity. Gold nanoparticles $(50 \mathrm{~nm})$ were uniformly embedded in amorphous carbon film on silicon substrate using methanol solution at the voltage of $1200 \mathrm{~V}$ for the deposition time of $5 \mathrm{~h}$ [33]. Under the same deposition conditions, the resistivity decreased from $108 \Omega \cdot \mathrm{cm}$ for the film without Au-doping to $104 \Omega$. cm with doped Au, which means that transition of amorphous carbon films from insulator to semiconductor should be also possible by doping metal nanoparticles.

Adding Palladium nanoparticles and using methanol and dehydrolinalool $\left(\mathrm{C}_{10} \mathrm{H}_{16} \mathrm{O}\right)$ as carbon source, the amorphous carbon film, in which Pd particles with a size of 1 - $5 \mathrm{~nm}$ evenly distributed can be successfully achieved [34].

\subsection{Non-Metal Dopants}

Doping nonmetal into DLC films is directed to enhancing the content of sp2 and improving optical and electrical properties. A low emission current greatly limits the application of amorphous films as cold cathode. Doping $\mathrm{N}$ into amorphous carbon films can elevate Fer$\mathrm{mi}$ level and lower work function, and then improve emission effect $[35,36]$. Carbamide and $\mathrm{C}_{60}$ derivant $\left(\mathrm{C}_{60}\right.$ $\left.\left[\left(\mathrm{NH}_{2}\right)_{2} \mathrm{CNCN}\right]_{5}\right)$ can be used as doping agent [37]. Typically, different $\mathrm{N}$ doping contents have been obtained from a mixed solution with various molar ratios of methanol to carbamide in the range of 1:2000 - 1:500 [38]. The deposition voltage fixed at $600 \mathrm{~V}$, the reaction time was $4 \mathrm{~h}$, and the reactor temperature was controlled at $60^{\circ} \mathrm{C}$. Analysis showed that nitrogen was doped into the film in the form of $\mathrm{C}=\mathrm{N}$ and $\mathrm{C}-\mathrm{N}$ bonds and the sp2 content increased. Meanwhile, the field emission current density came to $59.5 \mu \mathrm{A} / \mathrm{mm}^{2}$ while electric field was about $24 \mathrm{~V} / \mathrm{mm}$, which fulfill requirement of field emission display on electronic emitter.

Doping phosphorus even with a small amount for example $1 \%$ can strongly decrease the resistivity by $6-7$ orders of magnitude [39], improve the critical density of field emission and band gap was also increased. Moreover, it has been found that P-doped amorphous carbon films decreased platelet adsorption rate and activity, inhibited blood coagulation, and then may improve biological compatibility [40].

Wan et al. [41] used a mixed solution of methanol and three phenyl phosphate with the molar ratio of 1000:1 to prepare P-doped carbon films. Phosphorus combined with carbon in the form $\mathrm{C}=\mathrm{P}$ bond and the graphitization degree of the films enhanced confirmed by XPS and Raman spectroscopy. The experiments of the field emission characteristics showed that the threshold electric field and current density for the undoped- and doped-P films were $12 \mathrm{~V} / \mathrm{mm}, 45.7 \mu \mathrm{A} / \mathrm{mm}^{2}$, and $9.5 \mathrm{~V} / \mathrm{mm}, 12.6$ $\mu \mathrm{A} / \mathrm{mm}^{2}$, respectively [42]. Similarly, the emission properties can be also improved by doping sulfur using carbon disulfide [43] or thiophene [44] as dopant.

For doping fluorine, 2,2,2-Trifluoroethanol

$\left(\mathrm{CF}_{3} \mathrm{CH}_{2} \mathrm{OH}, \mathrm{TEF}\right)$ is a good candidate dopant [45], being identical in structure and property with methanol [46]. After fluorine doping, the amorphous carbon film is present as nano-scale bamboo shoots and the structure is closely packed and highly preferred orientation. The contact angle of the F-doped film with water is $145^{\circ}$. This is attributed to its special structure and low surface energy of the $\mathrm{CF}_{x}$, which is confirmed that fluorine exists in main forms of $\mathrm{CF}_{2}$ and $\mathrm{C}=\mathrm{CF}_{x}(x=1,2)$ in the film by infrared analysis.

\subsection{Other Dopants}

In order to decrease internal stress simultaneously on the premise of ensuring hardness, the allotropes of carbon including $\mathrm{C}_{60}$ and carbon nanotube were doped into DLC films. Hu et al. [47] had doped $\mathrm{C}_{60}$ nano particles into the amorphous carbon films. The concentration of $\mathrm{C}_{60}$ nano particles in the electrolyte was $0.28 \mathrm{mg} \cdot \mathrm{mL}^{-1}$. Deposition voltage, temperature, and reactive time were $1600 \mathrm{~V}$, $50^{\circ} \mathrm{C}$, and $10 \mathrm{~h}$, respectively. The result showed that the film hardness although little changed, the internal stress was effectively reduced.

Similarly, carbon nanotube is also easily doped in DLC films using liquid phase deposition. A typical experimental condition is that $\mathrm{N}, \mathrm{N}$-dimethylformamide (DMF, 99.5\%) is used as electrolyte, in which the content of carbon nanotube is $0.53 \mathrm{mg} \cdot \mathrm{mL}^{-1}$, and deposition voltage, 
temperature, and deposition time are $1400 \mathrm{~V}, 50{ }^{\circ} \mathrm{C}$, and 5 h, respectively [48]. As is known to all, carbon nanotube possesses excellent mechanical properties including high elastic modulus and toughness because of its special structure [49]. The DLC film after doping carbon nanotube has changed in property. The internal stress decreased from 1.2 GPa to $0.83 \mathrm{GPa}$, hardness increased from 10.28 $\mathrm{GPa}$ to $12.47 \mathrm{GPa}$, and Young modulus decreased from 253.64 GPa to 206.66 GPa. The spectrum analysis showed that the ratio of $\mathrm{sp} 2$ in the film increased.

Zhang et al. [50] prepared lamellar nano oxidized graphene from liquid phase, and then doped them into DLC film, where methanol used as reactant and the content of the oxidized graphene was $1 \mathrm{mg} / \mathrm{ml}$. Deposition voltage and time were $1200 \mathrm{~V}$ and $10 \mathrm{~h}$, respectively. The properties of the DLC films are obviously improved by doping graphite. The hardness and Young modulus are greatly increased from $5.1 \mathrm{GPa}$ and $137.4 \mathrm{GPa}$ to 10.1 GPa and 171.1 GPa, respectively, and the friction coefficient is also decreased significantly. The electrical resistivity decreases from $108 \Omega \cdot \mathrm{cm}$ to $102 \Omega \cdot \mathrm{cm}$. The explanation could be the inhibition of plastic deformation and the relaxation of internal stress by the lamellar grapheme [51].

An attempt has been made to dope $\mathrm{ZrO}_{2}$ into the carbon films in our recent research work under low voltage. In the experiments a mixed solution of acetic acid, alcohol, and deionized water with a volume ratio of 1:4:5 is selected as electrolyte. The presence of acetic acid can provide free hydrogen ions in abundance and alcohol is helpful to get a better suspension with nano $\mathrm{ZrO}_{2}$ particles. Raman spectra of the films deposited at a pulsed voltage of $100-200 \mathrm{~V}$ and a deposition time of 30min show that there are $\mathrm{ZrO}_{2}$ characteristic strong peaks located between $200-700 \mathrm{~cm}^{-1}$ and a broad peak centered at $1580 \mathrm{~cm}^{-1}$, attributing to amorphous carbon as showed in Figure 3. The film doped $\mathrm{ZrO}_{2}$ nano particles is very smooth and compact, as shown in Figure 4.

\section{Existing Problems}

Liquid electro-deposition technique is a promising method to synthesis amorphous carbon films on the complicated workpiece with large area under low temperature. However, there are still some problems to be solved.

1) Mechanical properties and adhesive strength

The recent researches focus on chemical components, morphology, and micro-structure. There is few report on the properties of the films deposited from electro-deposition because they are poor due to lack of high-energy ion bombardment during the film growth. There is an obviously difference in mechanical properties with the films by vapor phase deposition [53], although some improvements have been made by doping metal and in-

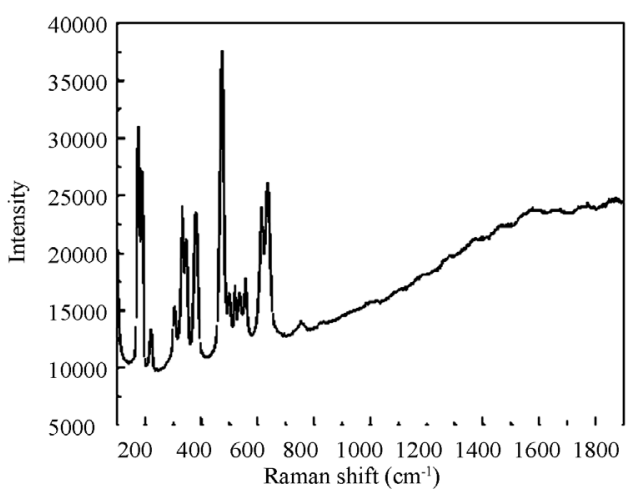

(a)

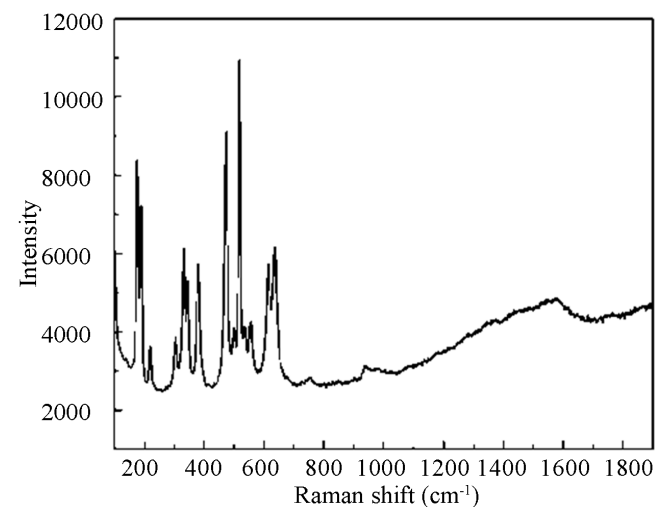

(b)

Figure 3. Raman spectra of the films doped with $\mathrm{ZrO}_{2}$ ((a) $0.6 \mathrm{mg} / \mathrm{L}$; (b) $0.2 \mathrm{mg} / \mathrm{L}$ ) [52].

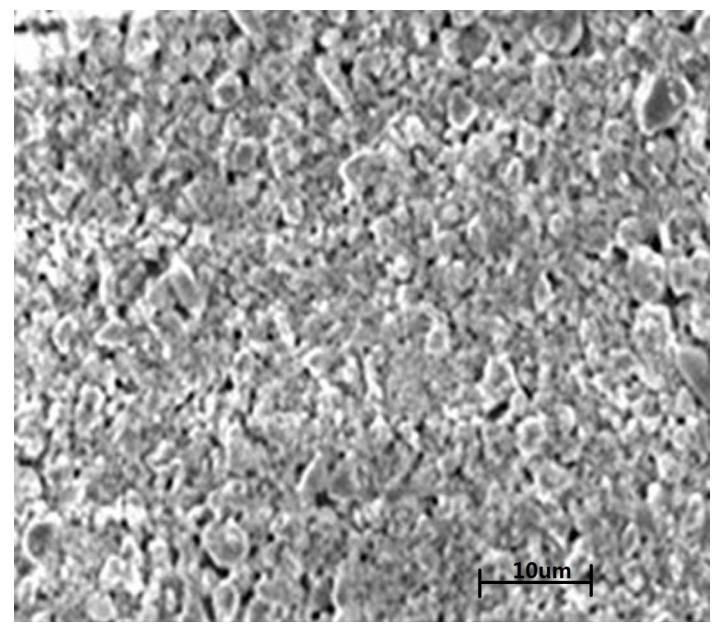

Figure 4. SEM photograph of the film doped with $\mathrm{ZrO}_{2}$ [52].

troducing additive agent. Table 4 shows the micro-hardness of the carbon films deposited using various deposition techniques.

2) The ratio of $\mathrm{sp}^{3} / \mathrm{sp}^{2}$

The properties of DLC films strongly depend on the ratio of $\mathrm{sp}^{3} / \mathrm{sp}^{2}$ [20]. The films deposited by liquid phase method contain too much graphite phase and their 
Table 4. Micro-hardness of the carbon films.

\begin{tabular}{cccc}
\hline Films type & $\begin{array}{c}\text { Preparation } \\
\text { method }\end{array}$ & $\begin{array}{c}\text { Vickers } \\
\text { hardness } \\
\left(\mathrm{Kg} / \mathrm{mm}^{2}\right)\end{array}$ & Reference \\
\hline a-C:H & r, f & $2000-4500$ & {$[17]$} \\
a-C:H & r, f & $3000-5000$ & {$[17]$} \\
a-C:H & Ion beam & $3000-5000$ & {$[17]$} \\
a-C:H & Magnet-Sputtering & $2500-4300$ & {$[17]$} \\
a-C:H & DC & -3000 & {$[17]$} \\
Dense Carbon & Ion beam & $\leq 6000$ & {$[17]$} \\
$\begin{array}{c}\text { DLC } \\
\text { nano-particles }\end{array}$ & Electro-deposition & $\sim 673$ & {$[38]$} \\
\hline
\end{tabular}

quality is difficult to control. It is necessary to try to find out suitable carbon sources, additive agents, and deposition process.

3) The exploration of deposition mechanism

Currently, study on the growth mechanism of carbon films from liquid phase deposition is still in the initial stage. Although some experimental results have been slightly analyzed, it is necessary to establish a perfect theory to guide experimental researches on film growth with high-quality.

\section{Prospects}

With the further research on growth of amorphous carbon films by electro-deposition, the theory and technology in this field will be gradually improved. The technique will possess a huge development potential and be widely used to prepare new materials because of unique properties and low cost. Research works in future should be more intensive in the following aspects: 1) selection of carbon sources, additives and dopants; 2) mechanism of formation and stability of amorphous or diamond-like carbon with diamond phase structure; and 3) application of the films.

\section{Acknowledgements}

The project is financially supported by the Cultivation Fund of the Key Scientific and Technical Innovation Project, Ministry of Education (N01707015) and by Major Project of Chinese National Programs for Fundamental Research and Development (2011CB013402).

\section{REFERENCES}

[1] J. Guo, H. Wang and H. Yan, "Recent Developments in the Preparation of Diamond-Like Carbon Films by the Liquid,” Chemistry Online, Vol. 7, 2007, pp. 521-526.

[2] L. I. Maissel and R. Glang, "Handbook of Thin Film Technology,” McGraw-Hill, New York, 1970.

[3] Y. Namba, "Attempt to Grow Diamond Phase Carbon
Films from an Organic Solution," Journal of Vacuum Science and Technology A, Vol. 10, 1992, pp. 3368-3370. http://dx.doi.org/10.1116/1.577829

[4] C. B. Cao, H. S. Zhu and H. Wang, "Electrodeposition Diamond-Like Carbon Films from Organic,” Thin Solid Films, Vol. 368, No. 2, 2000, pp. 203-207. http://dx.doi.org/10.1016/S0040-6090(00)00765-3

[5] M. C. Tosin, A. C. Peterlevitz, G. I. Surdutovich and V. Baranauskas, "Deposition of Diamond and Diamond-Like Carbon Nuclei by Electrolysis of Alcohol Solutions," Applied Surface Science, Vol. 144-145, 1999, pp. 260-264. http://dx.doi.org/10.1016/S0169-4332(98)00808-3

[6] R. S. Li, B. Liu, M. Zhou, Z. X. Zhang, T. Wang, B. A. Lu and E. Q. Xie, "Effect of Deposition Voltage on the Field Emission Properties of Electrodeposited DiamondLike Carbon Films,” Applied Surface Science, Vol. 255, No. 9, 2009, pp. 4754-4757. http://dx.doi.org/10.1016/j.apsusc.2008.10.053

[7] T. Paulmier, J. M. Bell and P. M. Fredericks, "Deposition of Nano-Crystalline Graphite Films by Cathodic Plasma Electrolysis,” Thin Solid Films, Vol. 515, No. 5, 2007, pp. 2926-2934. http://dx.doi.org/10.1016/j.tsf.2006.08.027

[8] Y. Y. He, G. F. Zhang, X. D. Hou and B. S. Cao, “Deposition of Diamond-Like Carbon Films from 2-Propanolby Liquid Electrochemical Technique,” Materials Review, Vol. 26, No. 6, 2012, pp. 90-92.

http://d.g.wanfangdata.com.cn/Periodical_cldb201212026 .aspx

[9] J. T. Jiu, K. Cai, Q. Fu, C. B. Cao and H. S. Zhu, "Liquid Deposition of Hydrogenated Carbon Films in N,N-Dimethyl Formamide Solution, "Materials Letters, Vol. 41, No. 2, 1999, pp. 63-66. http://dx.doi.org/10.1016/S0167-577X(99)00104-4

[10] N. Mayama, H. Yoshida, T. Iwata, K. Sasakawa, A. Suzuki, Y. Hanaoka, et al., "Characterization of Carbonaceous Films Deposited on Metal Substrates by LiquidPhase Electrodeposition in Methanol," Diamond \& Related Materials, Vol. 19, No. 7-9, 2010, pp. 946-949. http://dx.doi.org/10.1016/j.diamond.2010.02.039

[11] K. Sreejith, J. Nuwad and C. G. S. Pillai, "Low Voltage Electrodeposition of Diamond Like Carbon Films," Applied Surface Science, Vol. 252, No. 2, 2005, pp. 296-302. http://dx.doi.org/10.1016/j.apsusc.2004.11.091

[12] T. Paulmier, L. I. Emadand, J. M. Bellb and P. M. Fredericks, "Characterization of Reaction Products and Mechanisms in Atmospheric Pressure Plasma Deposition of Carbon Films from Ethanol,” Journal of Materials Chemistry, Vol. 15, 2005, pp. 300-306. http://dx.doi.org/10.1039/b409099c

[13] A. I. Kulak, A. I. Kokorin, M. Dieter, V. G. Ralchenko, A. V. Kondratyuk, et al., "Electrodeposition of Nanostructured Diamond-Like Films by Oxidation of Lithium Acetylide," Electrochemistry Communications, Vol. 5, No. 4, 2003, pp. 301-305. http://dx.doi.org/10.1016/S1388-2481(03)00050-X

[14] H. Q. Jiang, L. N. Huang, S. J. Wang, et al., "Synthesis of DLC Films by Electrolysis Dimethyl Sulfoxide," Electrochemical and Solid-State Letters, Vol. 7, No. 11, 2004, pp. D19-D21. http://dx.doi.org/10.1149/1.1807531 
[15] H. S. Zhu, J. T. Jin, Q. Fu, H. Wang, et al., “Aroused Problems in the Deposition of Diamond-Like Carbon Films by Using the Liquid Phase Electrodeposition Technique,” Journal of Inorganic Materials, Vol. 17, 2002, pp. 571-578.

[16] J. A. Dean, “Lange’s Handbook of Chemistry,” 2nd Edition, J. F. Wei, Trans, Science Press, Beijing, 2003.

[17] G. F. Zhang, J. Y. Du, Y. Y. He, G. Q. Li and X. D. Hou, "Surface Morphology of Diamond-Like Carbon Films Prepared by Liquid Deposition,” Journal of Chinese Electron Microscopy Society, Vol. 26, No. 1, 2007, pp. 19-23.

[18] Y. Li, G. F. Zhang, Y. Y. He and X. D. Hou, "Electrical Double Layer Model and Thermodynamic Coupling for Electrochemically Deposited Hydrogenated Amorphous Carbon Films," Journal of the Electrochemical Society, Vol. 159, No. 12, 2012, pp. 918-920. http://dx.doi.org/10.1149/2.034212jes

[19] S. Gupta, R. K. Roy, B. Deb, S. Kundu and A. K. Pal, "Low Voltage Electrodeposition of Diamond-Like Carbon Films,” Materials Letters, Vol. 57, No .22-23, 2003, pp. 3479-3485.

[20] S. Gupta, M. P. Chowdhury and A. K. Pal, "Synthesis of DLC Films by Electrodeposition Technique Using Formic Acid as Electrolyte,” Diamond \& Related Materials, Vol. 13, No. 9, 2004, pp. 1680-1689. http://dx.doi.org/10.1016/j.diamond.2004.02.006

[21] V. P. Novikov and V. P. Dymont, "Synthesis of Diamond-Like Films by an Electrochemical Method at Atmospheric Pressure and Low Temperature,” Applied Physics Letters, Vol. 70, No. 22-23, 1997, pp. 200-202. http://dx.doi.org/10.1063/1.118355

[22] P. Aublanc, V. P. Novikov, L. V. Kuznetsova and M. Mermoux, "Diamond Synthesis by Electrolysis of Acetates," Diamond and Related Materials, Vol. 10, No. 3-7, 2001, pp. 942-946.

[23] E. Matiushenkov, E. Shevchenko, D. Kochubey, D. Sviridov, A. Kokorinc and A. Kulak, "Synthesis of Carbon Films with Diamond-Like Structure by Electrochemical Oxidation of Lithium Acetylide," Chemical Communications, 2001, pp. 317-318.

[24] Y. Li, G. F. Zhang, X. D. Hou and D. W. Deng, "Synthesis and Tribological Properties of Diamond-Like Carbon Films by Electrochemical Anode Deposition,” Applied Surface Science, Vol. 258, No. 17, 2012, pp. 6527-6530. http://dx.doi.org/10.1016/j.apsusc.2012.03.070

[25] Y. Li, G. F. Zhang, X. D. Hou and D. W. Deng, "Growth Mechanism of Carbon Films from Organic Electrolytes," Journal of Materials Science, Vol. 48, No. 9, 2013, pp, 3505-3510.

[26] J. Robertson, "Diamond-Like Amorphous Carbon,” Material Science and Engineering Reports, Vol. 258, No. 37, 2002, pp. 129-281. http://dx.doi.org/10.1016/S0927-796X(02)00005-0

[27] S. H. Wan, L. P. Wang and Q. J. Xue, “An Electrochemical Strategy to Incorporate Iron into Diamond Like Carbon Films with Magnetic Properties," Electrochemistry Communications, Vol. 11, 2009, pp. 99-102. http://dx.doi.org/10.1016/j.elecom.2008.10.037
[28] H. Q. Jiang, L. N. Huang and Z. J. Zhang, "Facile Deposition of Copper-Doped Diamond-Like Carbon Nanocomposite Films by a Liquid-Phase Electrochemical Route,” Chemistry Communications, Vol. 7, 2004, pp. 2196-2197. http://dx.doi.org/10.1039/b408497g

[29] L. N. Huang, H. Q. Jiang and J. S. Zhang, "Synthesis of Copper Nanoparticles Containing Diamond-Like Carbon Films by Electrochemical Method," Electrochemistry Communications, Vol. 8, 2006, pp. 262-266. http://dx.doi.org/10.1016/j.elecom.2005.11.011

[30] J. Y. Zhang and Y. L. Yu, "Electrodeposition and Characterization of Pd Nanoparticles Doped Amorphous Hydrogenated Carbon Films," Solid State Sciences, Vol. 11, 2009, pp. 1929-1932.

http://dx.doi.org/10.1016/j.solidstatesciences.2009.07.012

[31] K. D. Ma. G. B. Yang, L. G. Yu, et al., "Synthesis and Characterization of Nickel-Doped Diamond-Like Carbon Film Electrodeposited at a Low Voltage," Surface and Coatings Technology, Vol. 204, 2010, pp. 2546-2550. http://dx.doi.org/10.1016/j.surfcoat.2010.01.039

[32] S. Hussain and A. K. Pal, "Synthesis of Composite Films of Mixed Ag-Cu Nanocrystallites Embedded in DLC Matrix and Associated Surface Plasmon Properties,” Applied Surface Science, Vol. 253, No. 7, 2007, pp. 3649-3657. http://dx.doi.org/10.1016/j.apsusc.2006.07.074

[33] G. Chen, J. Y. Zhang and S. R. Yang, “A Novel Method for the Synthesis of Au Nanoparticles Incorporated Amorphous Hydrogenated Carbon Films," Electrochemistry Communications, Vol. 9, 2007, pp. 1053-1056. http://dx.doi.org/10.1016/j.elecom.2006.12.019

[34] Y. L. Yu, S. Liu and J. Y. Zhang, "Cathode Electrodeposition and Characterization of $\mathrm{Ru}$ Nanoparticles Doped a-CNx:H Composite Films,” Diamond and Related Materials, Vol. 19, 2010, pp. 661-664. http://dx.doi.org/10.1016/j.diamond.2010.02.028

[35] D. S. Mao, J. Zhao and W. Li, "Electron Field Emission from Nitrogen-Containing Diamond-Like Carbon Films Deposited by Filtered Arc Deposition,” Materials Letters, Vol. 41, No. 3, 1999, pp. 117-121. http://dx.doi.org/10.1016/S0167-577X(99)00115-9

[36] A. A. Eytikh, H. Hartnagel and V. G. Litovchenko, "Enhancement of Electron Field Emission Stability by Nitrogen-Doped Diamond-Like Carbon Film Coating," Semiconductor Science and Technology, Vol. 19, 2004, pp. 923-929. http://dx.doi.org/10.1088/0268-1242/19/7/025

[37] Y. L. Yu and J. Y. Zhang, "Ultrafast Electrodeposition of Amorphous Carbon Nitride Films from Fullerene Derivative,” Electrochemistry Communications, Vol. 12, 2010, pp. 390-393. doi.org/10.1016/j.elecom.2010.01.001

[38] R. S. Li, E. Q. Xie, M. Zhou, Z. X. Zhang, T. Wang and B. A. Lu, "Field Emission Properties of Nitrogen Incorporated DLC Films Prepared by Electrodeposition,” Applied Surface Science, Vol. 255, 2008, pp. 2787-2790. http://dx.doi.org/10.1016/j.apsusc.2008.08.010

[39] J. Yuan, V. S. Veerasamy, G. A. J. Amaratunga, W. I. Milne, K. W. R. Gilkes, M. Weiler, et al., "Nitrogen Doping of Highly Tetrahedral Amorphous Carbon,” Physical Review B, Vol. 48, No. 24, 1993, pp. 17954-17959. http://dx.doi.org/10.1103/PhysRevB.48.17954 
[40] S. C. H. Kwoka, J. Wanga and P. K. Chu, "Surface Energy, Wettability, and Blood Compatibility Phosphorus Doped Diamond-Like Carbon Films," Diamond \& Related Materials, Vol. 14, No. 1, 2005, pp. 78-85. http://dx.doi.org/10.1016/j.diamond.2004.07.019

[41] S. H. Wan, H. Y. Hu, G. Chen and J. Y. Zhang, "Synthesis and Characterization of High Voltage Electrodeposited Phosphorus Doped DLC Films," Electrochemistry Communications,” Vol. 10, 2008, pp. 461-465. http://dx.doi.org/10.1016/j.elecom.2008.01.003

[42] S. H. Wan, L. P. Wang, J. Y. Zhang and Q. J. Xue, "Field Emission Properties of DLC and Phosphorus-Doped DLC Films Prepared by Electrochemical Deposition Process," Applied Surface Science, Vol. 255, 2009, pp. 3817-3821. http://dx.doi.org/10.1016/j.apsusc.2008.10.061

[43] S. Kundoo, P. Saha and K. K. Chattopadhyay, "Electron Field Emission from Nitrogen and Sulfur-Doped Diamond-Like Carbon Films Deposited by Simple Electrochemical Route," Materials Letters, Vol. 58, No. 30, pp. 3920-3924. http://dx.doi.org/10.1016/j.matlet.2004.08.018

[44] S. H. Wan, L. P. Wang and Q. J. Xue, "Electrochemical Deposition of Sulfur Doped DLC Nanocomposite Film at Atmospheric Pressure," Electrochemistry Communications, Vol. 12, No. 1, 2010, pp. 61-65. http://dx.doi.org/10.1016/j.elecom.2009.10.036

[45] J. Y. Zhang, G. Chen and S. G. Yang, "Fabrication of Hydrophobic Fluorinated Amorphous Carbon Thin Films by an Electrochemical Route," Electrochemistry Communications, Vol. 10, 2008, pp. 7-11. http://dx.doi.org/10.1016/j.elecom.2007.10.006

[46] H. Y. Hu, G. Chen and J. Y. Zhang, "Synthesis of C60 Nanoparticle Doped Hard Carbon Film by Electrodeposition,” Carbon, Vol. 46, 2008, pp. 1095-1097. http://dx.doi.org/10.1016/j.carbon.2008.03.012

[47] H. Y. Hu, G. Chen and J. Y. Zhang, "Facile Synthesis of CNTs-Doped Diamond-Like Carbon Film by Electrodeposition," Surface and Coating Technology, Vol. 202, 2008, pp. 5943-5946. http://dx.doi.org/10.1016/j.surfcoat.2008.06.162

[48] Z. J. Zhang, S. S. Fan, J. L. Huang, et al., "Pulsed Laser Deposition and Physical Properties of Carbon Nitride Thin Films," Journal of Electronic Materials, Vol. 25, No. 1, 1996, pp. 57-61. http://dx.doi.org/10.1007/BF02666174

[49] J. Y. Zhang, Y. L. Yu and D. M. Huang, "Good Electrical and Mechanical Properties Induced by the Multilayer Graphene Oxide Sheets Incorporated to Amorphous Carbon Films,” Solid State Sciences, Vol. 12, 2010, pp. 11831187.

http://dx.doi.org/10.1016/j.solidstatesciences.2010.03.017

[50] C. G. Lee, X. D. Wei, J. W. Kysar and H. James, "Measurement of the Elastic Properties and Intrinsic Strength of Monolayer Graphene,” Science, Vol. 321, 2008, pp. 385-388. http://dx.doi.org/10.1126/science.1157996

[51] N. K. Xu, A. C. Yin, G. F. Zhang and X. L. Zheng, "Hardness Mesurements of DLC Films,” Mechanical Science and Technology, Vol. 16, 1997, pp. 1063-1070.

[52] Y. Li, "Amorphous Carbon Films Deposited by Cathodic and Anodic Deposition Modes," Dalian University of Technology, Dalian, 2013, pp. 24-27.

[53] G. H. Chen and Y. H. Lu, "Hardness of DLC Films Deposited on Stainless Steel," Journal of Inorganic Materials, Vol. 11, No. 4, 1996, pp. 635-649. 